\title{
Anticipating the adoption of IoT in everyday life
}

\author{
Paul Coulton, Adrian Ioan Gradinar and \\ Joseph Galen Lindley
}

\begin{abstract}
Realising the potential economic and societal benefits of emerging and future technologies such as the Internet of Things (IoT) is dependent on a critical mass of potential users adopting them, this is often driven by whether users consider them to be acceptable. However, the processes that drive adoption and acceptability are rarely taken into consideration when researching emerging and future technologies. More often than not, either adoption is regarded as something that will naturally occur once technology is made available to the market, or the process of adoption is considered to be someone else's future work. The result is that the discovery of challenges and barriers to adoption and acceptability occur only after potentially problematic design patterns have become established and concretised at the core of devices and services. This, in turn, can result in even the most mundane designs having unintended consequences or compromised impact.
\end{abstract}

In this chapter we focus on IoT connected products which are often referred to as 'smart' in our IoT-enabled 'smart homes'. The espoused promise of the smart home is that it will make our lives easier by giving us more free time, improving our energy consumption, and saving money. However, one factor which is frequently absent from these discussions is the tsunami of data which is generated and collected as we add millions of IoT products and services to our home networks. While the nuance of the emergent Human-Data relationships may not be of immediate concern to the majority of their users, when this significant activity is unexpectedly brought to the fore it can challenge our expectations and perceptions of personal privacy in our homes. Such disruptions to notions of privacy then unbalance our perception of IoT devices' acceptability, causing users to either resist the adoption of new devices or potentially reject devices which had previously been adopted.

Addressing this challenge requires new approaches to design for future IoT products and services. The underlying Human-Data relationships need to become legible. Moreover, new ways of allowing potential users to experience such futures before problematic aspects are introduced are equally important. This brings future adoption and acceptability concerns to the centre of current design challenges. To this end, in this chapter, we present several examples of projects which utilised the Design Research method of Design Fiction, to explore these challenges. This research is based on work conducted within the PETRAS Cybersecurity of the Internet of Things Research Hub to explore critical issues in privacy, ethics, trust, 
reliability, acceptability, and security and the Objects of Immersion research project, The Living Room of the Future.

\section{Adoption}

A popular lens on adoption is Gartner's much-referenced hype cycle (Figure 10.1). Whilst the hype cycle has scant empirical foundation in any specific domain or context [1], its familiarity to researchers and commercial innovators alike, coupled with the compelling rhetoric such diagrams provide relating to technology research and development, makes it a highly useful figurative aid for conceptualizing adoption of technology [1]. The hype cycle places technologies on a graph of time (x-axis) against expectations and/or exposure (y-axis).

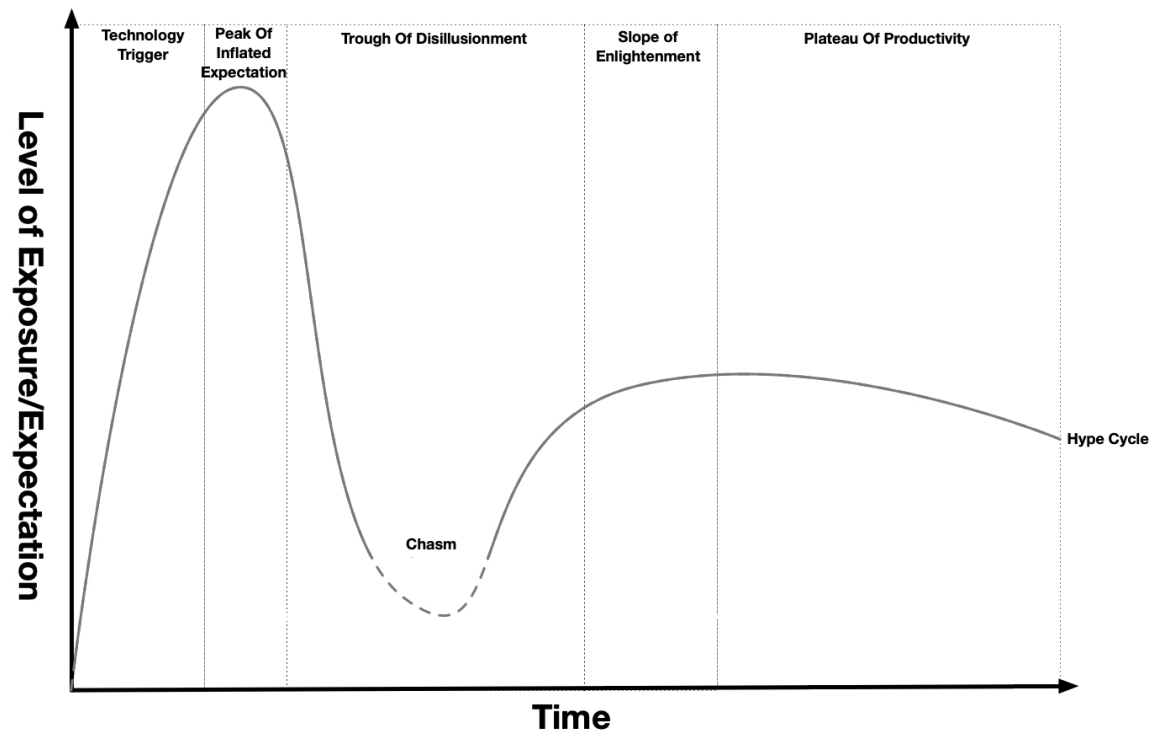

Figure 10.1 Adoption Hype Cycle

Starting from a particular technology the exposure and expectations grow quickly to a peak of inflated expectation - the fever pitch when early adopters and the media are very excited about the potential of a new innovation. This initial hype quickly falls away leading into a trough of disillusionment - the sad reality that most new technologies don't work immediately, and the adoption process can be drawn out. Exposure and expectations then grow again, this time more slowly, up the so-called slope of enlightenment - this is the actual, wide-scale, adoption. Finally, the technology stabilizes at the plateau of productivity - at this point a technology is no longer new, it is normal, or 'domesticated'. The so-called 'chasm', which sits at the bottom of the trough of disillusionment, was added to the diagram at a later stage to account for technologies which never make it up the slope of enlightenment - for example the Sinclair C5 personal transportation system. It's also the case that, sometimes, technologies - even if they make it across 
the chasm - actually jump back to re-enter the peak of inflated expectation such as Virtual Reality (VR) or Artificial Intelligence (AI).

Bell \& Dourish highlighted that 'we' (technology researchers) have a penchant for suggesting that the actual adoption of the technologies we research is 'just out of reach' or 'around the corner', and that considering how this is achieved, or possible unexpected consequences, is left as someone else's problem [2]. This is problematic because if we wait to see what potential societal impacts emerge before we consider how to address them, the challenge is much harder, and is often intractable as the issues are 'locked in' to the underlying technology and how it is used [3]. Of course, researchers are not able to fully envisage the gamut of potential futures for emerging technologies but this doesn't mean we shouldn't try. This leaves us with the question of how do we effectively explore future adoption?

\section{Technology Futures}

Considering the future is generally seen as an integral part of any design activity:

"Visions of the future are particularly important for designers, because designers have to imagine both the future conditions that will exist when their designs actually come into use and how those conditions will be changed by the creation of their new design" [4].

The general approach to envisioning futures has been to present futures through scenarios based on qualifiers, the most common qualifiers being probable, plausible, possible, represented in Figure 10.2. In some cases we might also add the preferable [5] as a moveable concept within any of the other qualifiers. The qualifiers are subjective but can useful be considered as: possible - might happen; plausible - could happen; and probable - likely to happen. Note the outer area of the cone could be considered the 'impossible' relating to concepts outside current scientific knowledge and if used would likely be considered fantasy.

We have not fully addressed the area of the figure indicating preferable. A preferable future could be described as what we believe should happen and thus could be applied within any of the three previously described qualifiers of probable, plausible, possible. This characterisation of preferable is potentially problematic because it effectively privileges the creator of the future scenario potentially promoting elitist views of a 'better world'. It should always be accompanied by a critical reflection on the question preferable to who, and why? 


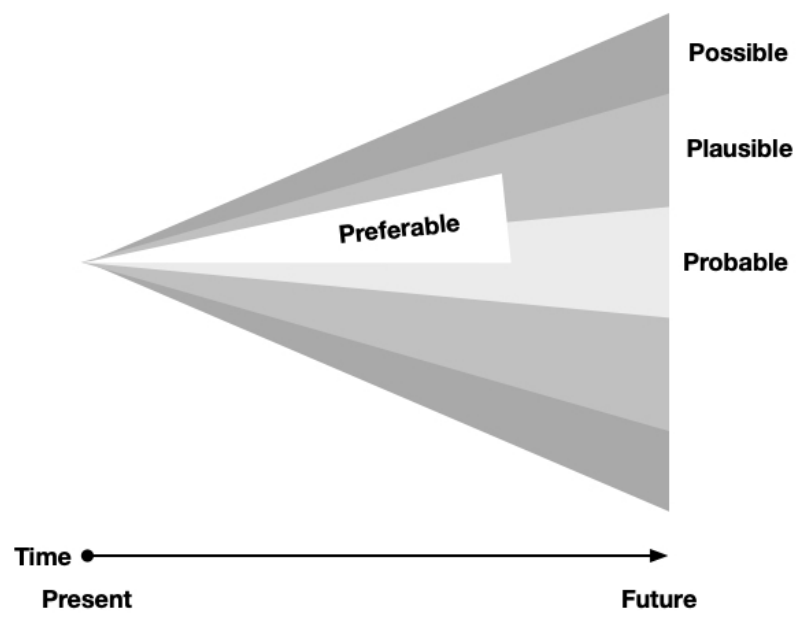

Figure 10.2. Futures Cone

Another problematic feature of the futures cone is how we acknowledge the influences of the past. Marshall McLuhan said in his book, The Medium is the Massage, "We look at the present through a rear-view mirror. We march backwards into the future" [6]. Although McLuhan meant this primarily as a criticism, it serves as a reminder of the significant influences of the past on our perception of both the present and the future. This is not to say we should completely ignore the past, as we may have lost potential futures through the decisions we made, but rather we should be aware of its influence. Further, we should acknowledge that there is no universally accepted view of the past, the present, or indeed the future (as Figure 2 suggests) - but rather these are individually constructed based on both reality and fiction [7] to create an ultimately particular reality [8]. This plurality of perspectives is vital as it also acknowledges the dominance of western visions in technology futures

"The notion of oww [One-World World] signals the predominant idea in the West that we all live within a single world, made up of one underlying reality (one nature) and many cultures. This imperialistic notion supposes the West's ability to arrogate for itself the right to be 'the world,' and to subject all other worlds to its rules, to diminish them to secondary status or to nonexistence, often figuratively and materially. It is a very seductive notion [...]" [9]

Rejecting $o w w$, and if we accept there are multiple simultaneous points of view then arguably what might be deemed plausible or probable can more meaningfully be collapsed into a single plausible qualifier. This prevents contention over the perceived difference, and reduces the chance that would overshadow otherwise productive discussions. With these points in mind we proposed an amended futures cone (Figure 10.3) which provides a more nuanced lens through which to consider futures [10].

Moving on from this discussion of the characterising futures we need a method through which we might practically consider future adoption of emerging 
technologies with various stakeholders. Whilst there are numerous methods proposed scenarios, sci-fi prototyping, etc in this chapter we are focussing on Design Fiction which is an approach the aims to concretize futures by considering what a world might be like in which emerging technologies have become domesticated into our everyday lives effectively rendering them as mundane as shown in Figure 10.4 (adapted from the work of James Auger [11]).

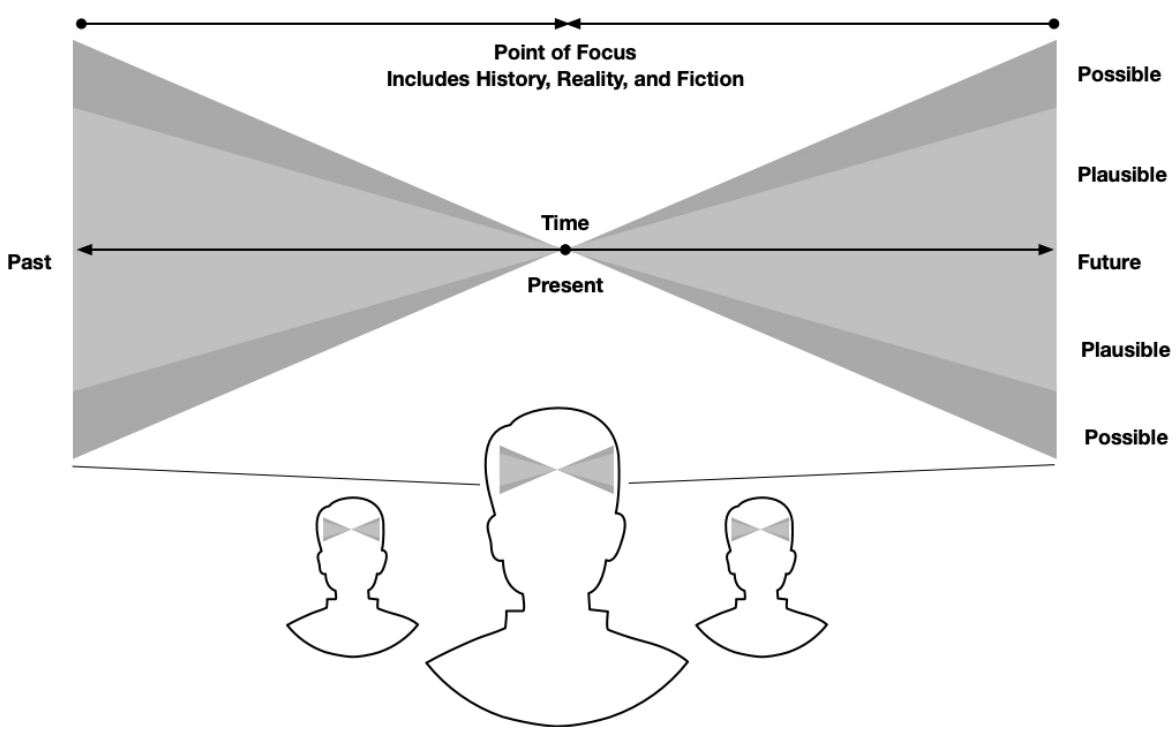

Figure 10.3. Plurality Futures Diagram

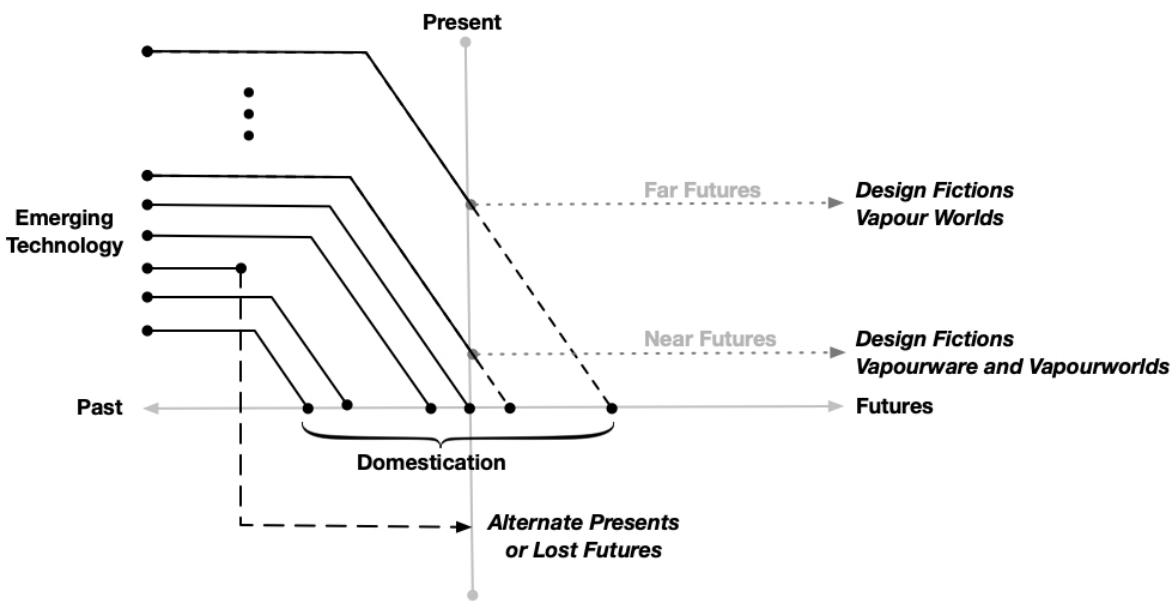

Figure 10.4. Emerging Technology Futures 
Unlike critical design which often presents such visions as dystopias using artefacts that are often designed to sit in exhibitions, galleries or museums, [11] Design Fiction appropriates formats more common in the real world. For example, product videos, device documentation, manuals, patents, media coverage, marketing materials, etc. In this way it evokes the traditions of vapourware and vapourworlds $[23,24]$ deployed by technology companies as representations of the future.

Vapourware is a term commonly used to describe software and hardware that is announced, sometimes marketed, but is never actually produced [12]. There are a variety of explanations as to why vapourware is produced such as unrealistic expectations of technology or over estimations of technical competence, but it has also been used nefariously by companies to inflate share prices, create extra publicity for their brand, or even deter competitors entering a market [23]. Vapourworlds is a term we use to describe material produced by commercial organisations which do not seek to promote a particular product but rather a future in which that company and its products are an integral component [23, 24].

Design Fiction draws upon these approaches and can help us to shift our gaze from the foreground of the present, to the horizon of the future, and then back again. Having glimpsed possible futures, Design Fictions helps us to consider both the broader range of societal implications of adoption and the wider political, regulatory, and social requirements that may be required for achieving adoption $[1]$.

\section{Design Fiction as Worldbuilding}

Design Fiction is still a relatively new concept within Design Research and still exhibits some traits of 'teenage angst'. In particular Design Fiction has had an identity crisis whereby there are currently a number of explanations of what it is, how it is produced, and what it is for. This means that there are currently competing understandings and framings of the practice resulting in ambiguity within any discussions produced about Design Fiction. To make our position clear within the context these examples we consider Design Fiction as a world building activity [13]. This means that the individual bits and pieces which make up a Design Fiction (the objects and artefacts produced by practice) are diverse and varied but the end result of Design Fiction is always the creation of a fictional world (13).

"Design Fictions are collections of artefacts that, when viewed together, build a fictional world. The artificially built world is a prototyping platform for the very designs that define it, meanwhile those designs reciprocate in kind and prototype the world." [13]

In practice, within a single Design Fiction, the specific selection of forms and media used manifest themselves as a number of standalone artefacts, which together build the world. Two metaphors are suggested for describing how the 
individual artefacts relate to the world. First, let us imagine a Design Fiction world as a distinct entity, one that we can see the overall shape of, but whose complex internal structure is hidden from view. What we can see, however, is a series of entry points into that world. Each artefact that contributes to making up the Design Fiction plays its role as a metaphorical entry point to the fictional world as shown in Figure 10.5.
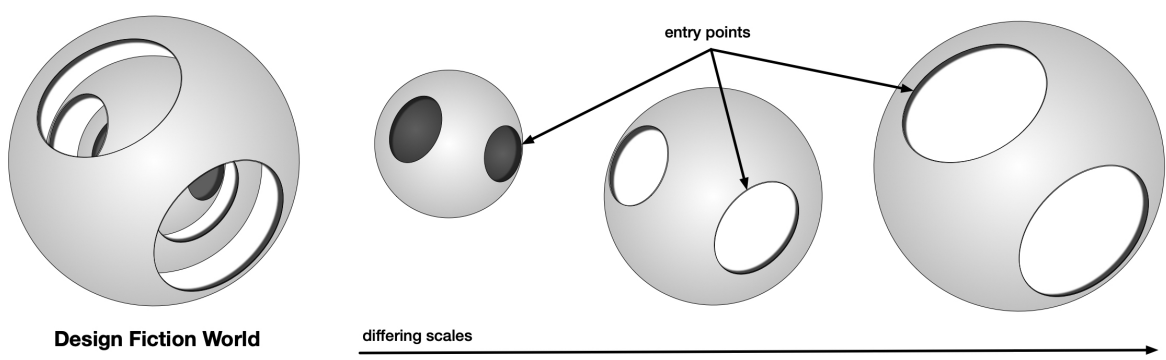

Figure 10.5. Design Fiction Worlds (scales and entry points)

The second metaphor, which works in unison with the first, is inspired by Charles and Ray Eames' iconic film about the relative size of things in the Universe, Powers of 10 (https://youtu.be/0fKBhvDjuy0). The film shows our world from a number of frames of reference (literally drawn as squares in the film) starting with a 1 meter squared section of an image that includes a couple sitting having a picnic, but then zooming out and increasing the visible area by one power of 10 every 10 seconds (e.g. 1 meter, 10 meters, 100 meters, 1000 meters, etc). This changing scale is a device that encourages the viewer to constantly reconsider the scene being viewed. It is important to note that by adopting this metaphorical framing we are not suggesting adherence to the configuration 1 power of 10 per 10 seconds, but rather that the basic concept of shifting scale can be applied to the consideration of Design Fiction worlds and the artefacts that create them. We can think of each individual artefact that constructs the world as a representation of that world, but at a different scale. The fictional world becomes a unique kind of prototyping platform testing and interrogating the very space artefacts which define it, meanwhile those artefacts return the favour and test and interrogate the world. It is through this back-and-forth prototyping relationship Design Fiction derives its value. In the following sections we will illustrate how Design Fiction can be utilised through specific projects to address the challenges of acceptability and adoption in the context of IoT technologies.

\section{Case Studies}

\subsection{Game of Drones}

Whilst the general focus for this chapter is predominantly how Design Fiction might address various challenges for IoT in the home i.e privacy ethics, security, trust etc. we start with a project the serves as an exemplar of creating Design Fictions. When we submitted Game of Drones as a 'work in progress' (WiP) paper [14], to the 'CHI Play' Conference in 2015, we wondered whether we would be 
derided or praised, because Game of Drones is a fictional account of a research project that never happened, presented in a real research paper submitted to a real conference. Whilst it may be surprising to some that such a paper passed review and was even accepted, it is important to understand the intent behind this paper was not subversive but rather to consider new ways to produce new knowledge [15]. The purpose of this project was dual: we wanted to explore a potential future use of drones for civic enforcement activities and also to progress a programme of research that aimed to develop Design Fiction as a research method. In this case study we want to illustrate the value of this approach by exploring how drones helped us develop a Design Fiction, and how Design Fiction helped us to highlight the wider issues relating to design of such a drone-based system.

The term 'drone' covers a broad range of unmanned aerial vehicles, but it is the proliferation of small quadcopters for personal use that has raised them to prominence in the public consciousness. Multi-rotor copters come with sophisticated flight controllers and on-board sensors which make them ever-easier to control. Their ease-of-use and relatively low cost have facilitated a huge range of controversial, sometimes amusing, and often innovative applications. For example, delivering drugs to prison inmates, a platform for espionage, flying cats and Halloween ghosts, a plethora of photographic applications, aerial light painting and competitive first-person view drone racing. Amazon's Prime Air, Facebook's internet drone, and a huge range of wildlife conservation drones are further examples of innovative commercial, corporate, and research uses of the same technologies. What these activities demonstrate is that while there is a high degree of 'interpretive flexibility' around drone technologies their full significance for society is still being established' as they start to be 'domesticated' and adopted into our everyday practices.

In Game of Drones, we were interested in exploring the potential use of dronebased civic enforcement systems. We wanted to understand what the technological and legal challenges of such a system would be, and to ask whether such a system would be an acceptable proposition and to initiate a discourse that could unpack the ethical and societal questions relating to such a system's adoption.

The Design Fiction world we built for Game of Drones was constructed using a variety of artefacts and then the whole 'world' was packaged into the submitted research paper. The WiP paper format provided the opportunity to focus on individual elements of the imaginary system and write about how they fitted together, as opposed to needing to create believable research results that would likely be expected of a full paper. The structure of the paper also allowed us to evaluate whether our world was plausible to the communities that may become involved in developing such technologies. By adhering to a normal paper structure we rendered a 'future mundane', in which drones had become an everyday part of the urban landscape. The paper describes a user trial of the Drone Enforcement System (DES) in which drones are used to provide enforcement services to local authorities. Specifically, it presents a 'gamified' system, which allows retired members of the police and armed services to act as remote drone pilots helping to enforce by-laws relating to parking offences and dog fouling in a small UK city. 
The whole interaction takes place through a game-like interface and points are awarded to pilots for recording activity, and ultimately, catching other citizens infringing upon the rules. Alongside the paper we submitted a supporting video (https://youtu.be/6b_30d7yW2s) which consisted of real footage recorded from a drone but was composited with a game-like interface and claimed - fictionally - to show the system 'in the wild'). In the following paragraphs we will discuss important elements that shaped the design of the system presented in the paper and video.

\subsubsection{Addressing the legality of Drones}

When the Design Fiction was created in 2015 the UK legislation the adoption of commercial services, such as the one described in Game of Drones, would be unfeasible because of Civil Aviation Authority Protocol 658 (Article 167) which governs small unmanned surveillance aircraft of less than $7 \mathrm{Kg}$ in weight. In particular, the article stipulates that, currently, drones must not be flown within 50 meters of any person, structure or vehicle and when flown via a First Person View (FPV) camera on the drone, they are required to adopt a 'Buddy Box' system whereby another person maintains a line of site view and can take over control of the drone if required. Within the realms of our fictional world, we therefore had to consider a future in which this article no longer applied and thus superseded Article 167 with our own law that allows remote FPV flight for users in possession of a 'Drone Pilot Proficiency Certificate' within certain height and distance limitations. Although only part of our more comprehensive Design Fiction world, this change in legislation is a Design Fiction prototype in its own right, and was arguably a portend of the US Federal Aviation Administration's subsequent implementation of compulsory drone registration and mandatory certification for commercial pilots and the recent additional restrictions imposed in the UK in relation to airports. This serves to highlight how design fiction can be used address policy or regulatory issues surrounding a particular technology and highlight potential barriers to adoption.

\subsubsection{Considering the required Infrastructure}

A number of technical details about hardware are included in Game of Drones, most notably; a contemporarily available consumer model of a drone and camera (cited as being used in the trial), as well as a sketch of the docking station design (Figure 10.6a), photographs of signage, and a diagram of the control device. While the drone hardware primarily played a supporting role in the Design Fiction, it allowed us to consider how current their current capabilities would impact upon the proposed system design. With this in mind the drone docking station was designed based on a real type of lamppost and would have been a necessary part of the infrastructure for this system due to the limited flight time of these battery powered vehicles. To further bolster the plausibility of the fiction we incorporated wireless charging technology (citing real research) into the docking stations and, because it would be necessary to make landing on the docking stations far simpler, we incorporated automatic landing beacons. We note that subsequent to creating our Design Fiction and publishing the paper, Amazon were awarded patent 
US009387928 in July 2016 which describes a remarkably similar lamppost-based docking station for drones to the one we proposed. Patents can be a useful way not only of representing possible futures but they can provide a useful source of information relating to potential futures as they effectively represent the desired futures of the companies that produce them.

\subsubsection{The devil is in the detail}

A number of extra details were added about the trial, all of which were intended to be highly reminiscent of reality, but with subtle twists for the Game of Drones world applied to them. These all add further to the Design Fiction's plausibility, but also draw upon cultural triggers to spark meaningful discussion. Most of us are familiar with parking or dog fouling notices, as these are oft-cited issues discussed by the public relating to their local urban environment, hence, we made real 'Drone Enforcement Zone' signs (figure 10.6b) that utilize the official UK designs for road signage. In addition, real GIS data was used to construct a map of the trial city, complete with enforcement zones (based upon existing city council maps of parking zones) and landing stations placed to facilitate coverage based on our flight distance restrictions and practical recharging as shown in Figure 10.7. Programmatically enforced no-fly zones are mentioned for special areas such as the railway line to acknowledge current discourses about the potential hazards caused by drones.

(a)

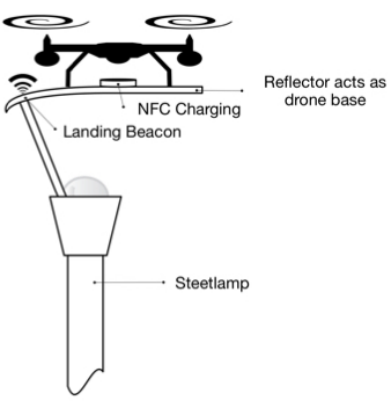

(b)
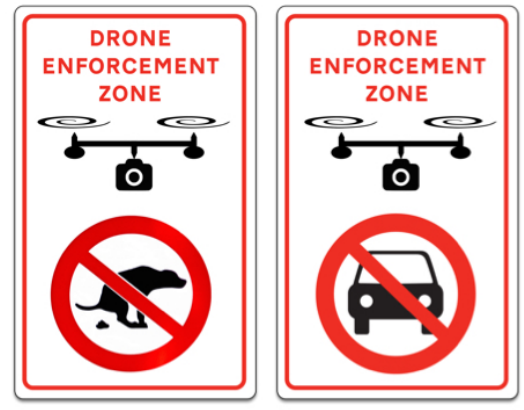

FBME OF DRONES

Figure 10.6. Drone Landing Station and Signage

Finally, the paper claims that the trial participants were ex-service (police and military) personnel, as they would likely have a diligent approach to enforcement. A note about financial rewards suggests that for this iteration of the system, users are not financially rewarded for recording more infringements, hinting at the possible ethical dilemmas of gamifying enforcement. It might seem that we went to a lot of trouble for such small details but we believe they add important texture to 
the fictional world, making it appear more plausible, and in doing so aim to stimulate more meaningful discourse.

For instance, "the drone pilots are also encouraged to record any activity they consider 'unusual' to ascertain the use of drones has potential for crime prevention beyond enforcement activities" [14] could suggest to some visions of a "big brother' style dystopia, while for others it may be reminiscent of Neighbourhood Watch schemes. This ambiguity is deliberate and done with the aim of encouraging discussion about the desirability of such systems and their effect on society.

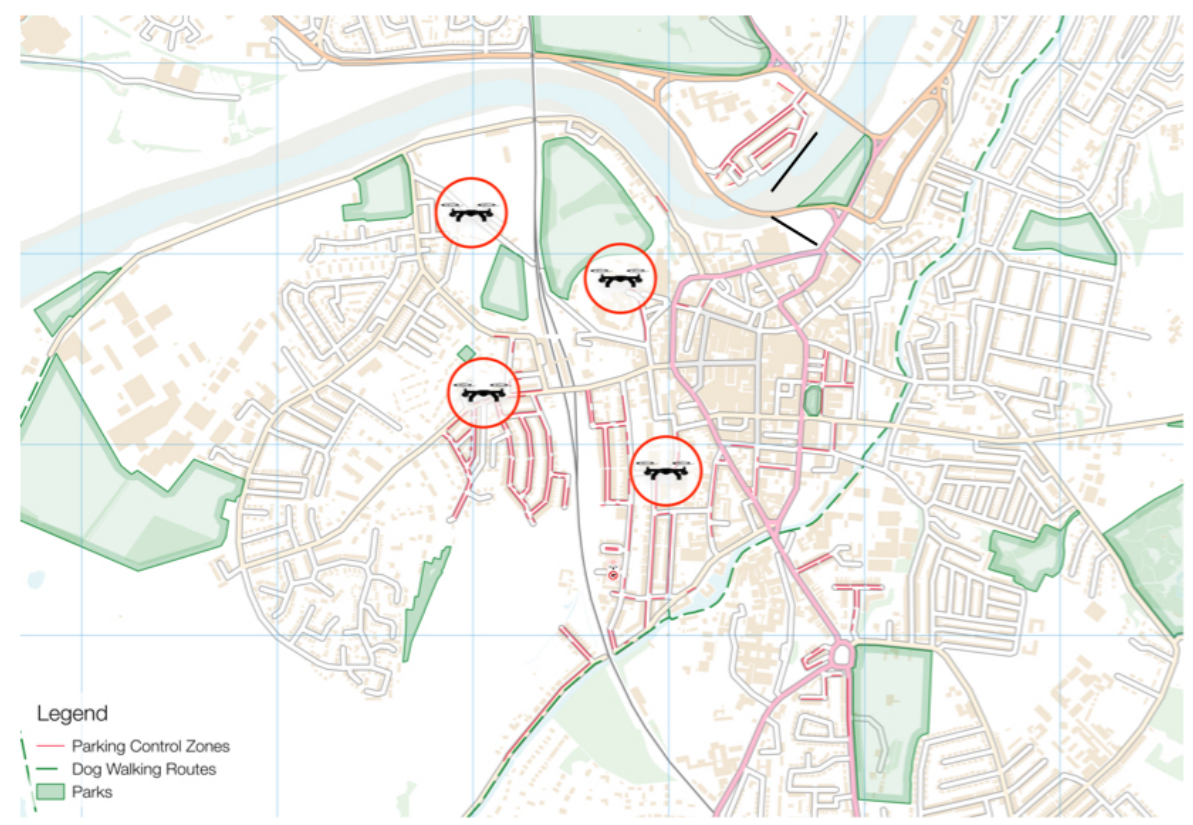

Figure 10.7. Map of Drone Landing Stations and Enforcement Zones

\subsubsection{Reflections}

Game of Drones built a fictional world in which drones were used as part of a gamified civic enforcement system. It did so convincingly enough that two out of three of the reviewers seemed to believe it was real despite our admission in the paper's conclusion that it was part of a Design Fiction, and therefore a 'fictional account' [15]. This level of acceptance, despite the evidence, is fascinating, not because of the misinterpretation but because it demonstrates how powerful technological visions of the future even to those shaping such futures (e.g. technology researchers). In this particular case not only is the proposed dronebased enforcement system plausible, it also seems to be feasible to the reviewers. Whilst at the time this form of civic based enforcement system seemed quite an extreme vision the recent Covid-19 has seen a rapid rise drone use from spraying disinfectant on streets, to enforcing lockdown in rural areas. Indeed the images from China of a Drone telling people to wear a mask and the footage from Derbyshire police shaming people walking their dogs in the UK Peak District 
national park are almost identical to scenes in the Game of Drones video. The aim of the Design Fiction was not to predict this future but rather highlight the potential future the emerging technology was leading towards in terms of wider adoption which we will explore for IoT in the following examples.

\subsection{Allspark}

This project explored a popular use case for IoT systems; intelligent energy infrastructures or 'smart grids'. The speculation was focused on a fictional energy company called Allspark. The Allspark project began not through a consideration of smart meters per se, which often appears in discussions of energy and IoT, but rather the somewhat neglected topic of batteries. Note this particular fiction does not address the important topic regarding the sustainability of IoT products themselves [16] or indeed batteries which present significant environmental challenges.

As our global electricity generation slowly moves towards sustainable sources, temporal energy storage is becoming an important element in ensuring our ability to cope with varying energy demand [17]. Although it is now quite common to generate electricity from wind and solar energy, the supplies are intermittent because the wind doesn't always blow and the sun doesn't always shine. Companies have started selling large batteries to install in your home that can store power generated when it is windy or sunny, and use it later at a time when it is needed. The same technology is beginning to be used on an industrial scale, which means that at peak times energy companies use huge arrays of batteries, which are charged by spare solar and wind energy. This avoids temporarily spinning up socalled 'Peaker' power plants, which run on polluting fossil-fuels. We wanted to use Design Fiction to explore the role the IoT might play in relation to how such battery technology and smart energy grids may manifest in next generation product design. We set out to explore the question: what if energy consumption could be optimised using batteries and the IoT, what would that world be like?

The initial research centred around electricity delivery systems and battery storage and highlighted a huge amount of wastage, particularly when electricity is converted from Alternating Current (AC) to Direct Current (DC) which has actually significantly increased over recent years. For example when power is generated by solar panels on the roof of a house is then normally converted to AC immediately, before either being used in the house or sent back to the grid. That conversion causes a loss of energy of approximately $30 \%$. It is also the case that the majority of our modern electronic devices use DC power, even though the power is delivered to the house using $\mathrm{AC}$, which requires conversion also resulting in a loss of energy. What if we could avoid some of the conversions? One way to do this, would be to redesign household electronic devices so that they would get their power from small rechargeable batteries, rather than from wall sockets. So, the first entry point into our Design Fiction was a universal, portable, rechargeable battery which would be used on an array of household products.

In a house equipped with the Allspark energy system, users would own multiple batteries and each one could be used to power a range of different devices. 
The smaller batteries would be charged directly from a much larger household battery (most households would have only one of these). The large batteries would usually be charged directly from solar panels on the roof of the house (and in some circumstances when there is spare capacity in the electricity grid). Because the solar panels, big batteries and small batteries, all operate using DC, there is a significant power saving when the system is configured like this. We began to imagine how these products would fit together and be marketed, naming the smaller 'utility' batteries Runner (Figure 10.8a) and the larger fixed batteries Director (Figure 10.8b) - drawing on terminology from the film industry.

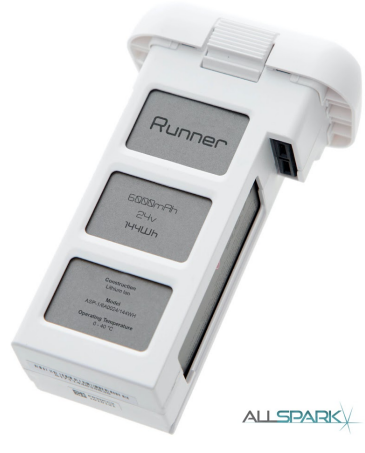

(a)

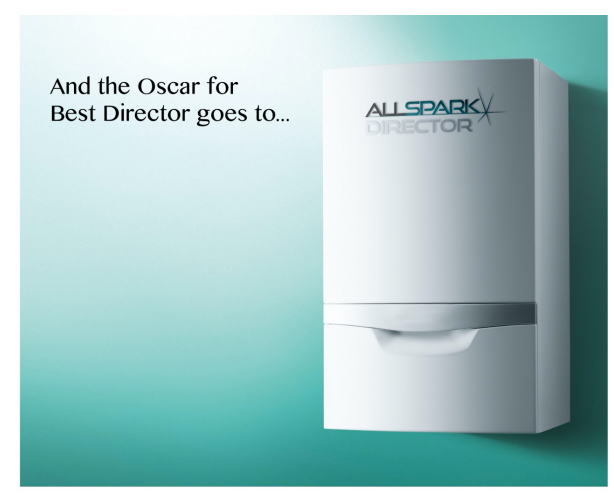

(b)

Figure 10.8: Allspark Battery Modules

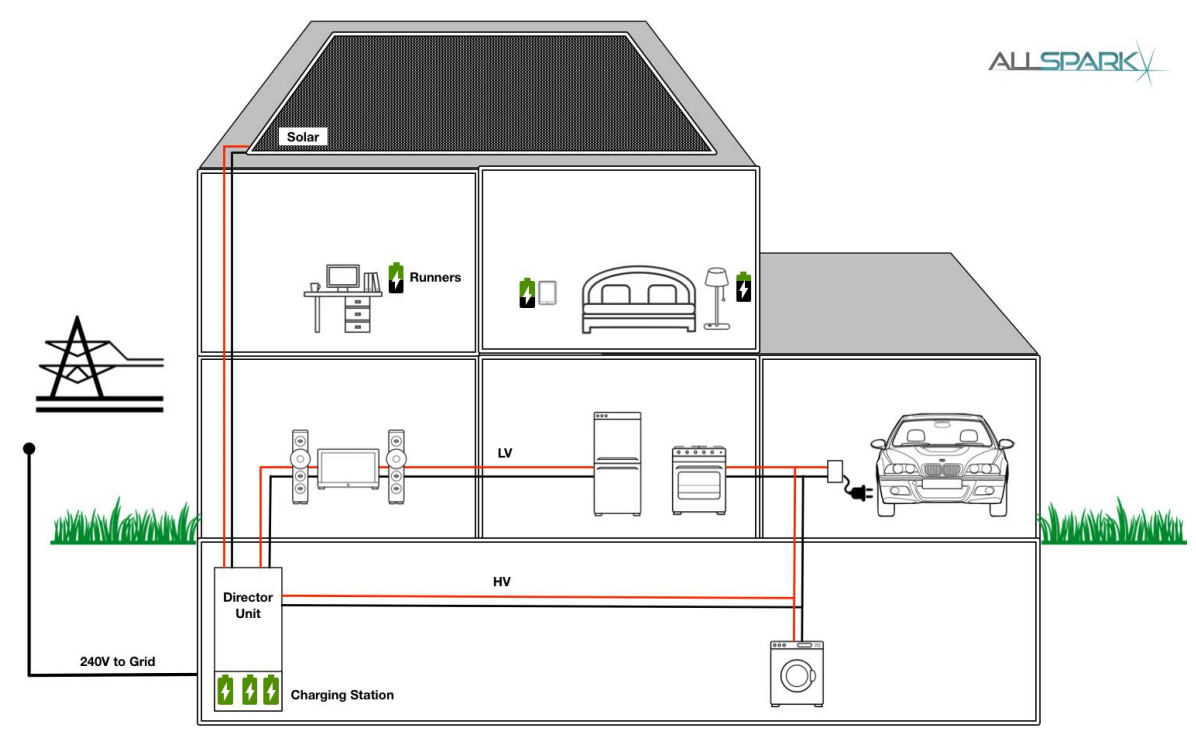

Figure 10.9: Alternative Home Wiring

In order to help visualise how the system would work in a typical home and how it would integrate with the existing power distribution network a further entry 
point (Figure 10.9) that shows a wiring schematic. Using the Allspark system, the main electricity grid remains the same - making this Design Fiction more plausible as it does not necessitate an entirely new infrastructure. However, within houses themselves many appliances would have to be replaced to either run off a newly installed high voltage DC circuit (e.g. the washing machine, cooker, and electric car) or from one of the Runner batteries (e.g. the laptop, tablet, or lamp). Further developing the 'zoomed out', or large scale, entry points for this Design Fiction, we produced fictional marketing materials as a way of thinking through what products could be powered from batteries (Figure 10.10a).

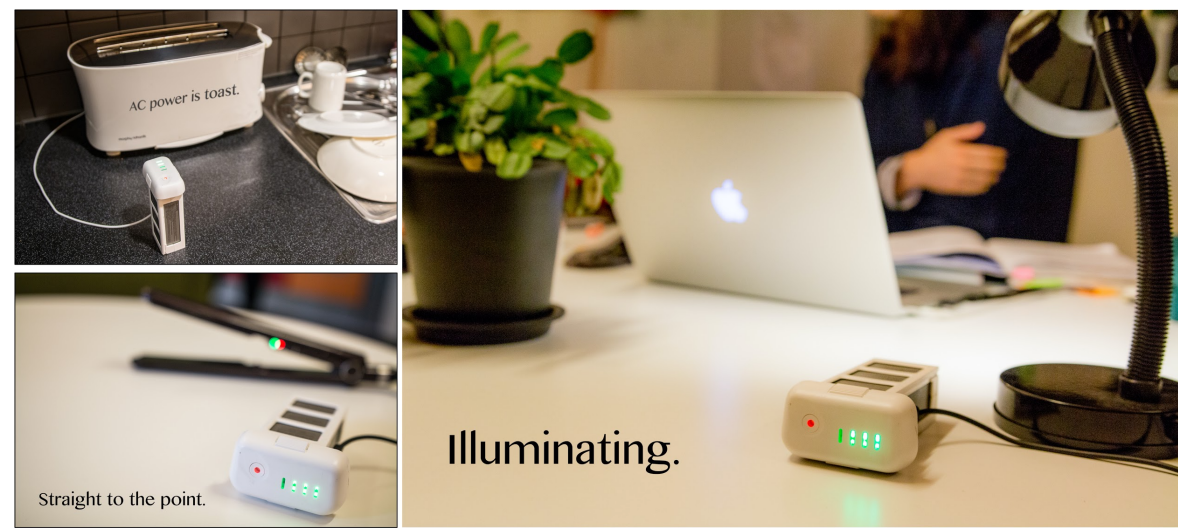

\section{Figure 10.10a: Allspark Promotional Material}

The entry points so far-Runner, Director, household wiring diagram, and advertising for battery-powered appliances-are effectively all zoomed out overviews. The more detailed entry points aroose through the consideration of the role the IoT could play in the Allspark system. Although a 'dumb' version of this system could bring efficiency to power consumption, to really optimise the system all of the elements need to be integrated. The energy grid operators would need to know how much energy is currently stored across every household's Directors and Runners and they need to know, as precisely as possible, how much energy will be used in the coming hours. By making every single Runner and Director battery an IoT device, it would be possible to gather the information necessary to drastically optimise electricity consumption, and take significant steps towards a renewablefirst grid.

In the Design Fiction world we created, the Allspark system would oversee internet-connected batteries across hundreds of thousands, or millions, of homes (Figure 10.10b).

Using the data provided by these smart batteries Allspark would be able to very accurately predict, and potentially manage, the demand. Ultimately Allspark could minimise the need for on-demand generation from fossil fuels. In a system like this it would be very likely that the operator, such as Allspark, would want to incentivise their customers to use devices at particular times. One way this could be achieved is with variable pricing, depending on supply and demand. The entry 
point below is more zoomed in than the others, and shows a screen in the Allspark app. Householders would utilise this app in order to plan their electricity usage around the price of electricity from the grid, how sunny it is, how many daylight hours there are, and how much charge there is in their battery system.

Through a 24 hour period we can see how the market price of electricity (if it is bought or sold from the grid) changes dramatically depending on various factors. During the night time (between sun down at 5.40pm and sun up at 7.36am) market price is at a high if winds are light. The app predicts that during this period the house should use battery power. Towards the end of the night, as the price of grid electricity goes down, the battery begins to charge from the grid. The app entry point (Figure 10.11a) helps us to understand not just how the price, charge and solar factors interact, but shows how the Allspark system might try to influence customer behaviour. It includes an option to select 'Nudge Aggression', which would impact upon how many notifications users get asking them to change their behaviour (an example is shown in the top-left of figure 11a, asking the user to turn off their air conditioner for 10 minutes in order to get a cost benefit of $£ 0.50$ ). 


\section{SPARKING THE INTERNET OF ENERGY

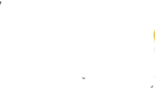

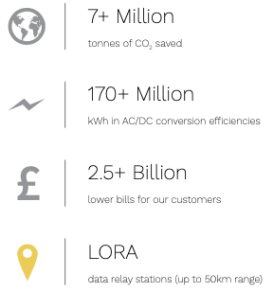

\section{DIRECTOR}

Charging towards optimisation

our rational network of Director energy storage appliances
untlise LORA wirreless technology to sthare real-time energy data for aligorthimic optim sation. Director is the hub of the Allspark system in your home and

\section{RUNNER}

Sock it to the socket

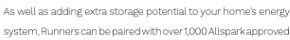

Directwhe applances that are aready on the market.
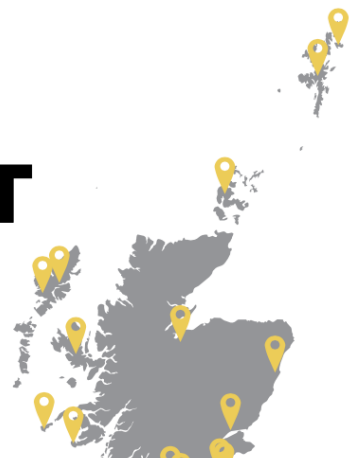
s.
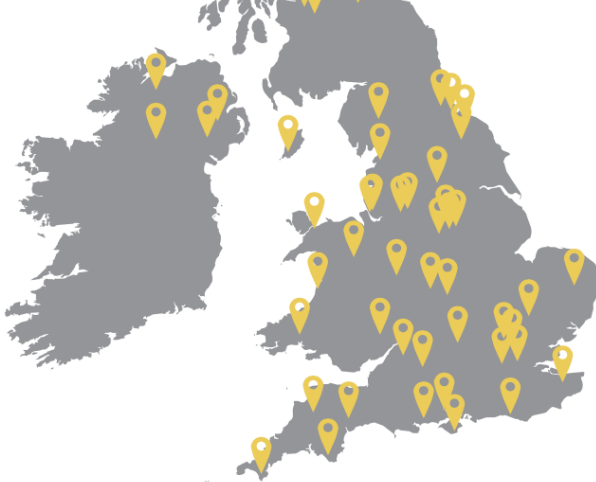

Figure 10.10b. Allspark Promotional Material

\section{DIRECTWIRE APPLIANCES}

Wash this space

With over 1,000 products already on the market, a vAT rebate
on your first three purchases, and appilances so capable they

required a brand new Eurcpean efficiency classification to be created this Directwire ecosystem is growing fast. 
The entry point shown in Figure 10.11b - an Allspark approved washing machine - shows how individual appliances would become integral parts of the whole system. Employing a basic nudge technique to try and influence behaviour towards more efficiency, appliances like washing machines would automatically schedule so that they run at a time that maximises available energy. Rather than indicating to the user how long is left before the wash is finished, it would display the latest possible time for when the wash would complete. We use the term 'energy temporality' to describe the way that a smart grid will likely have a knockon effect on how we use and interact with our electrical appliances and may ultimately require a change in design criteria. For example, if appliances are to be used at night making them quiet might be an increased priority.

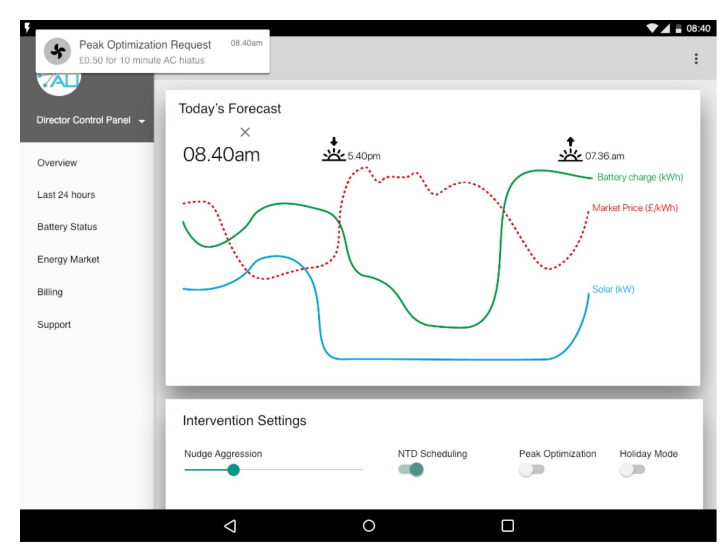

(a)

(b)

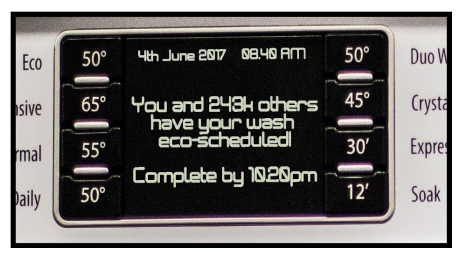

Figure 10.11. Allspark Feedback

\subsubsection{Reflections}

This particular manifestation of Design Fiction could be used by product designers to develop their understanding of how future energy distribution will impact upon their designs, alternatively it could be a useful tool for energy companies that need to refresh our energy infrastructure, lastly it may be used as a communication and policy tool to demonstrate how to increasing potential customers adoption by by making IoT more meaningful. Using Design Fiction in this way leverages the power of showing precisely what specific strategies for meeting medium-term emission targets look like, and in doing so aims to contribute towards negotiating acceptable IoT devices with an increased chance of adoption.

\subsection{Living Room of the Future}

In this case study we highlight the flexibility of the Design Fiction approach through the Living Room of the Future (LRofTF), which we characterise as an Experiential Design Fiction [18]. The LRofTF mixes both real and fictional elements to allow us to situate the audience in a near future world in which the negotiability of data access is brought to the fore. The LRofTF explores this by looking at how media broadcasters may utilise the potential of a technology called Object-Based Media (OBM) to deliver more immersive experiences to audiences in 
home environments. OBM allows the customisation of media, like radio and television, based on audience-specific data. For example, it may customise a soundtrack based on your music preferences. OBM delivers personalised viewing experiences by considering the programme as a collection of smaller elements created during production (these are referred to as media objects) and describing how they need to semantically relate to each other in order for the programme's flow or narrative to make sense. This system allows the media objects, in theory, to be dynamically reassembled into many possible personalised versions of the same programme. In addition to using OBM, the LRofTF uses IoT devices and external data sources to personalise the media even further, for example, by using the current weather to alter how the screen media is displayed, or using personal data to create customised sound tracks for programmes as shown in Figure 10.12. Finally, the IoT objects provide a physical means to contribute to an immersive media experience; for instance, the smart lights may adjust their colour and brightness automatically to match the overall look and feel of the content being shown on the screen.

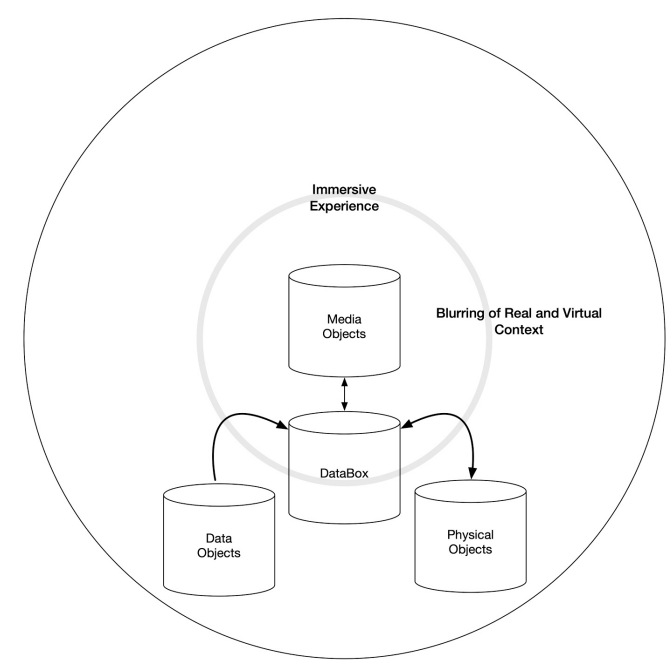

Figure 10.12: Relationship of Object Types for Living Room of the Future 

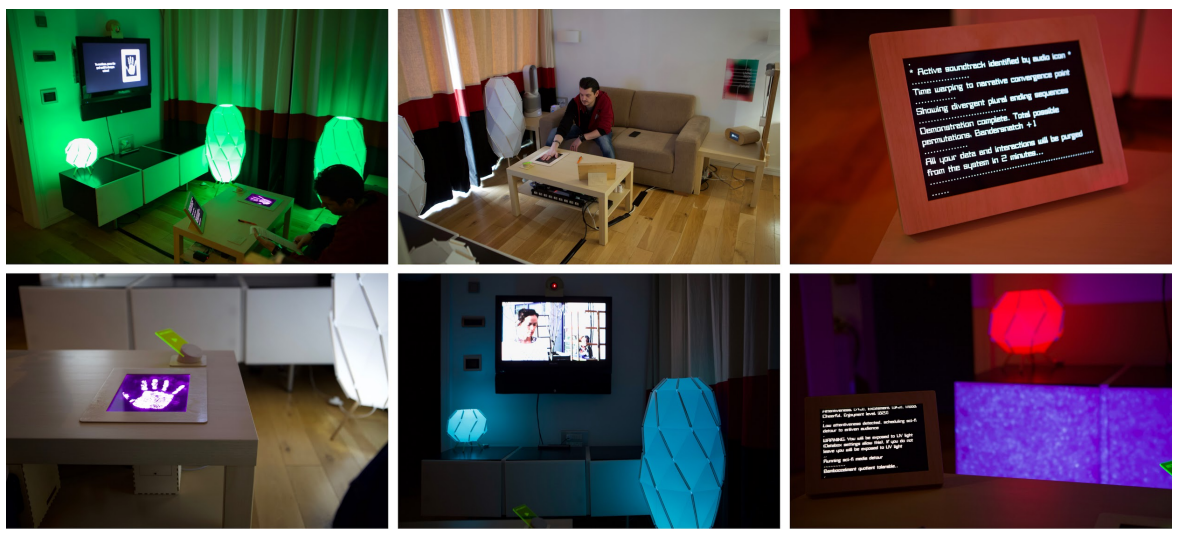

Figure 10.13: Living Room of the Future Installation

We chose to build this prototype around a living room, since this is a well understood space within our own homes which many people in the UK (who were our primary audience) are familiar with. Whilst the initial version of the LRofTF was designed as part of a public exhibition for a specific installation in the FACT gallery in Liverpool (UK) [19], it has been redesigned with new interactions and a new narrative for events at the Victoria and Albert Museum and the Tate Modern in London before going on permanent display in a 'Future Home' at the Building Research Establishment in Watford as shown in Figure 10.13. In the next sections, we describe the physical, media and data objects which sit at the foundation of delivering this experiential future. We have chosen to make this separation in writing to provide a greater level of understanding however, it is important to note they were not conceptualised in this manner, but rather holistically to provide a unified experience.

\subsubsection{Physical Objects}

The LRofTF was designed to represent a potential near future, and we identified a selection of off-the-shelf IoT products to form part of the experience, which included programmable lights, a heating/cooling fan, programmable window blinds, and smart plugs. In addition to these commercially-available products, we created a set of bespoke artefacts: a clock-radio whose speaker provides ambient sounds as part of the media; a series of sensors to detect audience interactions with objects in the room (including a drink coaster and a remote-control device); a coffee table with in-built hand sensor, visual display, and thermal printer; and a voice activated LED 'eye' which provides a personality for the living room and acts as the sensor for its face-scanning technology. Whilst the commercial products and the printer may be considered as 'outputs' of the LRofTF, the sensors should be seen as 'inputs' that generate data the system then uses to personalise the experience.

\subsubsection{Media Objects}


The version of the LRofTF described in this case study uses a short drama called The Break Up, specially commissioned by the BBC's Research and Development department to highlight the potential of dynamic programming for television. The Break Up is a special type of programme which utilises an innovation known as 'Perceptive Media' which had previously been demonstrated for radio [20]. Perceptive Media, in contrast to interactive storytelling (e.g., the Netflix Black mirror episode Bandersnatch) which relies on audiences to influence a storyline through direct action, utilises contextual information relevant to audiences passively, and data gathered using a range of sensors, to subtly alter the media. Although the overall story arc remains the same for each viewing, ambient aspects of the narrative tweak the ways media are presented in order to create a more engaging, context-specific, experience for the audience.

The Break Up was written and filmed in such a way that the entire narrative can be shifted to accommodate the viewer. For example, there are two contrasting endings (one positive, one negative) and two paths through the story (one emphasising the male character, and one highlighting the female). There is even an alternate version of the story, using the same script, where the gender roles of the characters are swapped, and rather than existing in the present day it takes place in a Science Fiction alternate universe - allowing for so-called 'genre spiking'. Further, it allows for dynamic changes of the soundtrack to better reflect each viewer's individual experience and tastes.

\subsubsection{Data Objects}

The media objects in the film can be reconfigured based on data objects which means that the system will construct a new version of the programme based on the contextual information about the viewer gathered through the available data points. This version makes use of real data points such as the current weather, location and time in addition to fictionally suggesting it uses data points such as music preferences, political leanings, social media postings, house heating habits, and metadata gathered from pictures. Taking the data points in conjunction with information gathered from the IoT sensors in the room (e.g. whether the audience is smiling, if they are moving around in their seats or whether looking away from the screen) and processed by the OBM system allows the delivery of a completely personalised version of the film. While the physical and media objects are the only tangible aspects, it is the data objects - despite being invisible - which directly affect how the audience experience LRoTF.

\subsubsection{What About Privacy?}

Given that each IoT device uses different protocols and shares data differently, we had to create a bespoke system for translating this data into a format that the LRofTF could understand. In order to address the privacy challenges of the experience (which acted as if it accessed various kinds of highly personal data) all data moving through the system is managed through an instantiation of Databox [21] providing a unique ecology for exploiting personal data in privacy-preserving ways. For example, Databox can enable a media provider to utilise algorithms that 
process data referring to an individual's viewing habits in order to offer bespoke content. However, uniquely, it can do so without disclosing personal data directly to the provider. Instead of distributing personal data to remote cloud servers for processing, processing takes place on-the-box, preserving privacy by ensuring no personal data needs to leave the home or be accessed remotely.

\subsubsection{The Experiential Future}

The Living Room of the Future experience is split into three parts: the introduction, the personalised media experience and the 'reveal', these parts are described in the following subsections providing an overview of design considerations.

\section{Introduction}

To begin, the participants seat themselves on the sofa in front of the television screen. The experience is then introduced by the voice of the living room which seeks to gain consent from users to collect, process and store their data (the LRofTF prints out a permission slip using the thermal printer embedded in the coffee table, which the audience must sign in order for the system to proceed). Based on the data collected the LRofTF then produces a unique version of the Break Up for that particular audience.

\section{Personalised Media Experience}

In the second part of the experience the film is played based on a profile generated by the system. During this film various IoT objects in the room begin to contribute to the experience. For example, at the start of the film the blinds come down and the room's lighting adapts to each scene (the system 'knows' the outside weather and picks up a relevant colour gradient). When the lead character in the film is outdoors, the fan switches on, matching the wind blowing her hair. The music within the film is chosen dependent on the profile generated by the system, as is the chosen ending (which depicts the character either leaving or staying with her abusive partner). By the middle of the story, if the audience appears bored (based on sensory inputs such as face scanning) an IoT smart plug is triggered by OBM to turn on an Ultra Violet (UV) light during which a short section of the science fiction film is shown to 'enliven' the audience, before returning to the main drama. The impact of particular data interactions which affect the drama do not immediately affect the media objects, which means that while each experience was uniquely tailored to the audience, they would not necessarily be able to see why or how. Therefore, the tablet in the coffee table highlights when data is being collected and subsequently used.

\section{Reveal}

Finally, as the audience has only experienced one of the many possible adaptations of the drama, an explainer video shows the variations which could have occurred, and why, as shown in Figure 10.14. At the end, the voice of the living 
room asks the audience to comment on their experience. In order to illustrate the audience reaction we filmed a number of consenting participants to create a GoggleBox style video (https://youtu.be/cjxbXbF_TPE) which provides a novel way of representing data related to the experience.

\subsubsection{Reflections}


The experience was designed to highlight the lack of control when dealing with providing consent and the generally unperceived transactional nature of data collection. Consent, even after the introduction of the General Data Protection Regulations (GDPR), often ultimately comes down to a binary choice of using the IoT service or not [22]. This is foregrounded through the living room's voice which will always keeps cajoling the audience for a positive acknowledgement to any consent questions even when the audience continually says 'no' the system says that this is a shame as they will miss out on the video but they are welcome to exit through the gift shop. It is interesting that during the hundreds of times the experience was run, only one person declined the fictional face recognition software scanning their face and when presented with the potential termination of the experience, the participant quickly changed their mind. This perhaps indicates how engaging voice can be as an interaction and a problem for future IoT systems using voice in that, if their security is compromised, they may present nefarious actants a highly effective means of phishing. 


\section{THE BREAK UP}
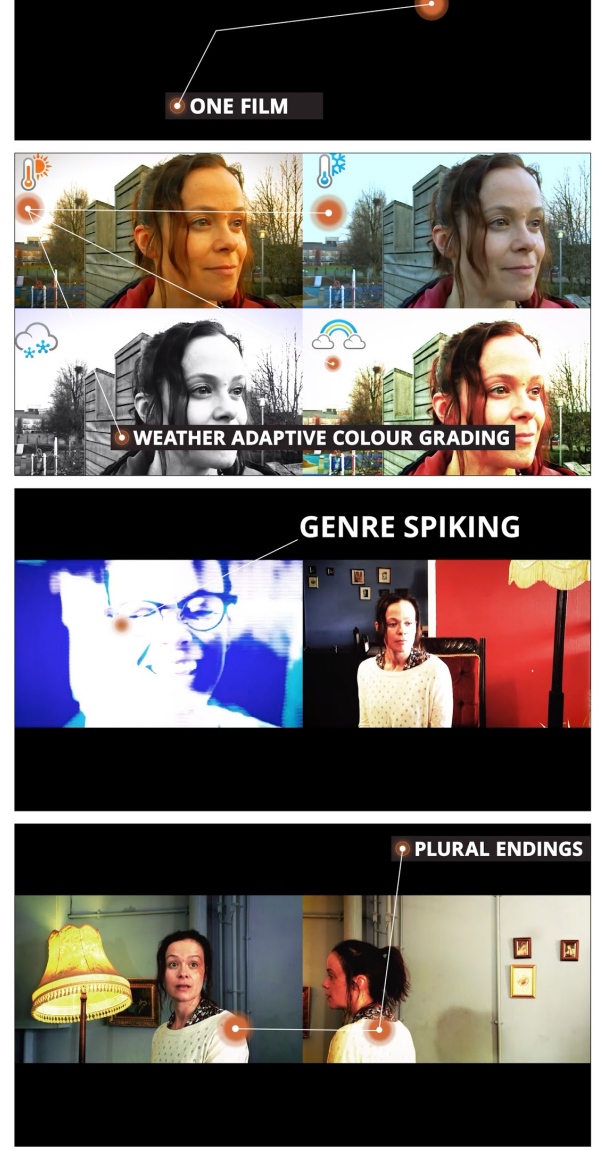

Figure 10.14: Break Up Explainer Video

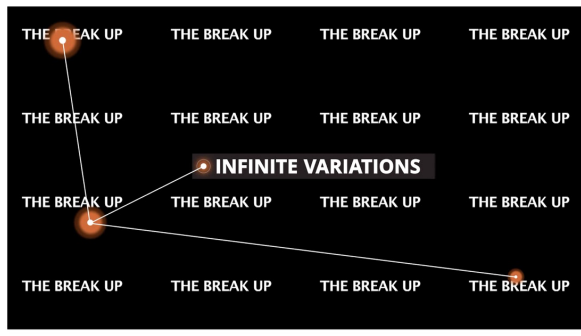

PLOT-DRIVEN SOUNDTRACKS
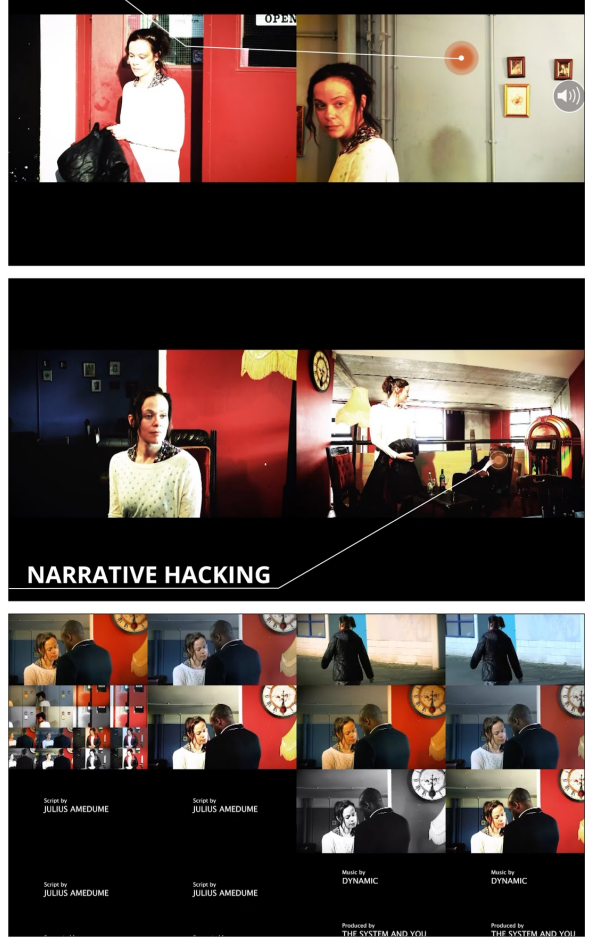
Alongside consent, LRoTF served to highlight the transactional nature of data and expose how often those transactions are generated simply by living in our homes. This was illustrated on the display and the thermal printer embedded in the coffee table. The display endeavoured to make data collection and how it was used legible by presenting this in real-time as the experience unfolded. This output onto a printed receipt for participants to take at the end of their experience to highlight that the data was also theirs if they wish to take greater control. The underlying questions being around the theme, how might transactional data practices impact acceptability and adoption?

\section{Conclusions}

As we stated at the start of this chapter, within research focussed around emerging technologies adoption IoT innovations is typically viewed as someone else's problem. This conundrum is thus forwarded into some other proximate future, despite an economic argument for adoption often being the driver of the very research agendas which fund innovation. This is potentially problematic as there may be significant societal and technological challenges that ultimately decrease the likelihood of adoption. Hence, the suggestion is that in some cases it is beneficial to incorporate a consideration of the wider societal and technical implications that could arise from particular instantiations of IoT technologies. In this work we have presented Design Fiction as Worldbuilding as a viable means to respond to these challenges. Though, notably, not as a way to predict future adoption as there will always be a plurality of potential futures, but rather to unpack what wider societal and technical challenges require consideration if future adoption is to be driven by acceptability.

\section{Acknowledgments}

The work featured in the chapter has been made possible through the PETRAS Cybersecurity of the Internet of Things Research Hub funded by the EPSRC, under grant EP/N023234/1, EP/N023013/1, and EP/N02317X/1, AHRC funded project Objects of Immersion AH/R008728/1, and EPSRC project Experiencing the Future Mundane EP/S02767X/1.

\section{References}

[1] Lindley J, Coulton P, Sturdee M. Implications for adoption. In Proceedings of the 2017 CHI Conference on Human Factors in Computing Systems 2017 May 2 (pp. 265-277). 
[2] Bell G, Dourish P. Yesterday's tomorrows: notes on ubiquitous computing's dominant vision. Personal and ubiquitous computing. 2007 Feb 1;11(2):133-43.

[3] Lanier J. You are not a gadget: A manifesto. Vintage; 2010.

[4] Berry DJ. Man-made futures: Readings in society, technology and design: edited by Nigel Cross, David Elliott and Robin Roy, The Open University Press, 1974.

[5] Voros J. A generic foresight process framework. foresight. 2003 Jun 1.

[6] Fiore Q, McLuhan M. The medium is the massage. New York: Random House; 1967.

[7] Gonzatto RF, van Amstel FM, Merkle LE, Hartmann T. The ideology of the future in design fictions. Digital creativity. 2013 Mar 1;24(1):36-45..

[8] Law J, Urry J. Enacting the social. Economy and society. 2004 Aug 1;33(3):390-410.

[9] Escobar A. Designs for the pluriverse: Radical interdependence, autonomy, and the making of worlds. Duke University Press; 2018 Mar 15.

[10] Coulton P, Burnett D, Gradinar AI. Games as speculative design: allowing players to consider alternate presents and plausible futures. In Lloyd P, Bohemia E, editors, Proceedings of Design Research Society Conference 2016. Design Research Society. 2016. p. 1609-1626. (Proceedings of DRS 2016). https://doi.org/10.21606/drs.2016.15.

[11] Auger J. Speculative design: crafting the speculation. Digital Creativity. 2013 Mar 1;24(1):11-35.

[12] Atkinson P. Delete: a design history of computer vapourware. Bloomsbury Academic; 2013 Oct 24.

[13] Coulton P, Lindley, J, Sturdee M, Stead M. Design fiction as world building. n: Proceedings of the 3rd Biennial Research Through Design Conference, 2017 March 22-23, Article 11, pp. 163-179. DOI: 10.6084/m9.figshare.4746964.

[14] Lindley J, Coulton P. Game of drones. In Proceedings of the 2015 annual symposium on computer-human interaction in play (CHIPlay). 2015 Oct 5 (pp. 613-618).

[15] Lindley J, Coulton P. Pushing the limits of design fiction: the case for fictional research papers. In Proceedings of the $2016 \mathrm{CHI}$ Conference on Human Factors in Computing Systems 2016 May 7 (pp. 4032-4043). 
[16] Stead M, Coulton P, Lindley J. Spimes Not Things: Creating A Design Manifesto For A Sustainable Internet of Things. The Design Journal. 2019 Jun 1;22(Suppl.

1):2133-2152.

https://doi.org/10.1080/14606925.2019.1594936

[17] Lindley JG, Coulton P, Cooper R. Not On Demand: Internet of Things Enabled Energy Temporality. In DIS '17 Companion Proceedings of the 2016 ACM Conference Companion Publication on Designing Interactive Systems. New York: ACM. 2017. p. 23-27 https://doi.org/10.1145/3064857.3079112

[18] Coulton, P, Lindley, JG, Gradinar, AI, Colley, J, Sailaja, N, Crabtree, A, Forrester, I \& Kerlin, L, Experiencing the Future Mundane. in Proceedings of RTD 2019. Research through Design 2019 , Delft, Netherlands, 19/03/19. https://doi.org/10.6084/m9.figshare.7855790.v1.

[19] Sailaja N, Crabtree A, Colley J, Gradinar A, Coulton P, Forrester I, Kerlin L, Stenton P. The living room of the future. InProceedings of the 2019 ACM International Conference on Interactive Experiences for TV and Online Video 2019 Jun 4 (pp. 95-107).

[20] Gradinar, A, Burnett, D, Coulton, P, Forrester, I, Watkins, M, Scutt, T \& Murphy, E 2015, Perceptive media: adaptive storytelling for digital broadcast. in Proceedings INTERACT 2015 The 15th IFIP International Conference on Human-Computer Interaction, Bamberg, Germany, 14-18 September.

[21] Mortier, R., Zhao, J., Crowcroft, J., Wang, L., Li, Q., Haddadi, H., Amar, Y., Crabtree, A., Colley, J., Lodge, T. and Brown, T., 2016, December. Personal data management with the databox: What's inside the box? In Proceedings of the 2016 ACM Workshop on Cloud-Assisted Networking (pp. 49-54). ACM.

[22] Lindley JG, Coulton P, Cooper R. The IoT and Unpacking the Heffalump's Trunk. In Arai K, Bhatia R, Kapoor S, editors, Proceedings of the Future Technologies Conference (FTC) 2018: Advances in Intelligent Systems and Computing. Vol. 880. Springer. 2018 https://doi.org/10.1007/978-3-03002686-8_11

[23] Coulton, P. and Lindley, J. (2017) 'Vapourworlds and Design Fiction: The Role of Intentionality', The Design Journal, 20. doi: 10.1080/14606925.2017.1352960.

[24] Coulton, P \& Lindley, JG 2016, Game vaporware as design fictions. in Proceedings of Mindtrek 2016. ACM, New York, pp. 341-349, Mindtrek 2016, Tampere, Finland, 17/10/16. https://doi.org/10.1145/2994310.2994313 
